\title{
Comparing Validity and Diagnostic Accuracy of Clarke's Angle and Foot Posture Index-6 to Determine Flexible Flatfoot in Adolescents: A Cross-Sectional Investigation
}

\author{
Fatma Hegazy (iD ${ }^{1,2}$ \\ Emad Aboelnasr ${ }^{2}$ \\ Mohamed Abuzaid (D) ${ }^{3}$ \\ In-Ju Kim (iD ${ }^{4}$ \\ Yasser Salem ${ }^{5}$ \\ 'Department of Physiotherapy, College of \\ Health Sciences, University of Sharjah, \\ Sharjah, United Arab Emirates; ${ }^{2}$ Faculty \\ of Physical Therapy, Cairo University, \\ Giza, Egypt; ${ }^{3}$ Department of Medical \\ Diagnostic Imaging, College of Health \\ Sciences, University of Sharjah, Sharjah, \\ United Arab Emirates; ${ }^{4}$ Department of \\ Industrial Engineering and Engineering \\ Management; College of Engineering, \\ University of Sharjah, Sharjah, United \\ Arab Emirates; ${ }^{5}$ Department of Health \\ Professions, School of Health Professions \\ and Human Services, Hofstra University, \\ Long Island, NY, USA
}

Objective: Clinically, the foot posture index-6 (FPI-6) and Clarke's angle (CA) are widely utilized to assess static foot posture; however, due to scarcity of scientific evidence, clinicians continue to debate the validity and diagnostic accuracy of these measures in children and adolescents. This study aimed to evaluate and compare the validity and diagnostic accuracy of the FPI-6 and CA in determining adolescents' flexible flatfoot between ages of 12 and 18 years, considering radiographic investigation as a reference standard measure.

Design: Cross-sectional study.

Setting: Governmental hospitals.

Participants and Methods: A sample of 460 people (920 feet) with flexible flatfoot between the ages of 12 and 18 (15.23 \pm 0.84 years) was enrolled in the study. The findings of the investigated measures were compared to the reference standard radiographic measure and plotted on the receiver operating characteristic (ROC) curve. The area under the ROC curve (AUC) was determined as a measure of FPI-6 and CA diagnostic accuracy. Intra-rater reliability, sensitivity, specificity, predictive values, and likelihood ratios of FPI-6 and CA were calculated and compared. The post-test probability of flatfoot was determined using the Fagan nomogram.

Results: CA had a substantially higher intra-rater reliability $(\mathrm{ICC}=0.99)$ than the FPI-6 (ICC $=0.96$ ), with p-value of 0.001 . CA has greater sensitivity (98\%) than FPI-6, as well as a superior specificity (99\%), positive predictive value (98\%), negative predictive value (98.9\%), positive likelihood ratio (97), and a lower negative likelihood ratio (0.02). CA had such an area under ROC curve of 0.98 with a 95\% confidence interval of $0.95-1.00$, while FPI-6 had an area under ROC curve of 0.80 with a $95 \%$ confidence interval of (0.77-0.85).

Conclusion: FPI-6 and Clarke's angle are both valid and diagnostically accurate clinical tests for flatfoot detection, with Clarke's angle outperforming FPI-6 in adolescents aged 12 to 18 years with a normal BMI.

Keywords: flatfoot, adolescents, Clarke's angle, foot posture index-6, validity, diagnostic accuracy

\section{Background}

The most prevalent foot deformity in children and teenagers is flexible flatfoot. It is distinguished by the collapse of the medial longitudinal arch (MLA) in the weightbearing position, which recovers to normal in the non-weight-bearing position. ${ }^{1}$
Department of Physiotherapy, College of Health Sciences, University of Sharjah, Sharjah, United Arab Emirates

Email fhegazy@sharjah.ac.ae 
There is a considerable debate as to which assessment measures are to be best included in flatfoot evaluation. ${ }^{2,3}$

It is commonly acknowledged that foot posture differs during the period of growth and musculoskeletal maturation, much like foot posture in children differs from the one in adolescence and in adulthood. ${ }^{4,5}$ There is an appreciable scarcity of the available literature published studies about foot posture in adolescence; however, adolescence is an important phase of one's life, marked by a various diversity of developmental, physiological, and anatomical adaptations. ${ }^{6}$ Abnormal foot posture in adolescence was found to be positively correlated with the development of a variety of functional problems, lower extremity musculoskeletal injuries, ${ }^{6-8}$ abnormal posture, or even a fullyfledged disability later in adulthood. ${ }^{9-11}$

Hence, studying the morphological and musculoskeletal changes in the foot, while making use of using valid, pertinent, reliable, and non-invasive clinical assessment tools throughout childhood and adolescence, is crucial for clinicians and researchers, in terms of understanding the atypical developmental trajectory of the foot throughout its successive variable stages of growth and development. ${ }^{12}$ The resultant body of this expertise knowledge would be expected to effectively support accurate diagnosis, proper decision-making, accurate intervention and subsequent therapeutic management.

Numerous measures are employed to diagnose flatfoot based on MLA height. ${ }^{1,3,13}$ Clinical assessment tools (FPI6 , measurement of navicular height, anthropometric measurements, and visual observation), ${ }^{1,14-17}$ radiological assessment, ${ }^{18}$ ultrasonography, photographic techniques, and footprint analysis, ${ }^{13,19,20}$ are all utilized to diagnose flatfoot based on MLA height.

Despite the fact that X-ray assessment is considered as the reference standard measure in the examination of static foot posture, ${ }^{1}$ its radiation exposure has significant drawbacks, particularly in the pediatric population. Moreover, the financial costs, as well as the need for professional staff to interpret radiographic findings, make it difficult to be used easily in clinical settings. Furthermore, under certain conditions such as screening a huge population in epidemiological studies, radiographic assessment of flatfoot may be irrelevant. ${ }^{3,21}$ In such cases, valid, safe, and noninvasive clinical assessment tools should be beneficial.

Footprint analysis and FPI-6 are the most widely used clinical methods for assessment of static foot posture, ${ }^{10,14}$ however, disagreement persists over whether footprint analysis can evaluate foot posture. Some studies even doubt whether a footprint can accurately represent $\mathrm{MLA}^{22,23}$ however, several investigations have shown that footprint indices correlate with radiographic measures. Kanatli et $\mathrm{al}^{24}$ reported that footprint analysis is as accurate as radiologic measurements, Villarroya et $\mathrm{a}^{25}$ found a significant correlation between $\mathrm{CA}$ and ChippauxSmirak index (CSI) methods and radiography-based talus-first metatarsal (T1MA) and calcaneal inclination angles.

$\mathrm{CA}$ is the most commonly used footprint parameter in clinical settings as it is a reliable tool and easy to be measured. ${ }^{15,26} \mathrm{CA}$ had been validated for assessing flatfoot in adults $(\geq 40 \text { years old })^{20}$ and in preschool children aged 3-6 years. ${ }^{15}$ However, these results cannot be generalized for children and adolescents because of the age-related changes in Paediatric foot with growth and musculoskeletal maturation. ${ }^{6}$

The systematic review proposed by Banwell et $\mathrm{al}^{1} \mathrm{did}$ not include $\mathrm{CA}$ as a valid measure for evaluation of paediatric flatfoot, the authors based their decision upon five studies [Nikolaiduou and Boudolos; ${ }^{27}$ Villarroya et al $;{ }^{25}$ Chen et al $;{ }^{15}$ Pauk et al; ${ }^{26}$ Pauk and Szymul] ${ }^{28}$ Only one study ${ }^{15}$ out of these five studies had investigated validity of CA in preschool children. While, the remaining four studies either investigated the reliability of $\mathrm{CA},{ }^{26}$ the association between foot structure and BMI, ${ }^{25}$ the difference between flatfeet and normal feet for foot type classification rational using footprint measures ${ }^{27}$ or compared vertical ground reaction force data between flat and neutrally aligned feet. ${ }^{28}$ Therefore, we cannot consider that $\mathrm{CA}$ is not a valid measure for pediatric flatfoot based upon the results of this systematic review, ${ }^{1}$ because of the paucity of the available literature about the psychometric properties (reliability and validity) of the studied clinical assessment measures to classify foot posture in paediatric population as concluded by the authors of this systematic review. ${ }^{1}$

The FPI-6 is an observational scoring system considering the three-dimensional nature of foot posture, ${ }^{29}$ with good reliability in adults ${ }^{30,31}$ and children. ${ }^{31-33}$ FPI-6 has been validated in adults ${ }^{34}$ and Paediatric population. ${ }^{14}$

Gijon-Nogueron et al, in $2019^{35}$ had concluded that compared with FPI-6, CA overestimates and misguides paediatric foot posture, while other researchers found CA to be a practical, reliable, and sensitive measure. ${ }^{15,20,36,37}$ However, the diversity of the methodologies used and the circumstances for each study makes comparisons between the results of these previous studies difficult, leading to an ongoing debate among researchers and clinicians. This 
was highlighted as a limiting factor in the previous systematic studies. ${ }^{1,3,38,39}$

One way to settle this debate about diagnostic accuracy of FPI-6 and CA in adolescents, through investigating validity and diagnostic accuracy of FPI- 6 and CA against one criterion standard measure under the same circumstances on the same sample of the population. Therefore, this study aimed to investigate and compare the validity and diagnostic accuracy of CA and FPI- 6 against radiographic findings as a criterion standard measure to determine flexible flatfoot in adolescents aged 12-18 years. Based upon the aforementioned information and the previous studies, ${ }^{1,35}$ the main hypothesis of this study was that FPI-6 is more valid and diagnostically accurate in determining flatfoot in adolescents.

\section{Methods}

\section{Study Design}

A cross-sectional study design had been followed.

\section{Participants}

A convenient sample of 460 participants (920 feet) aged 12-18 years (mean age \pm standard deviation (SD) of 15.23 \pm 0.84 years) was recruited from the outpatient orthopedic clinic, Ministry of Health Hospital, Sharjah, UAE from September 2019 to May 2020.

Based on a clinical evaluation by an orthopedic surgeon who specializes in the foot and ankle, participants were diagnosed with bilateral flexible flatfoot. Physical examination, as reported by Gould ${ }^{11,40}$ was used to diagnose and classify Flexible pes planus. The patient's arch height and hindfoot valgus were evaluated with his/her back to the physician and then again from standing on toes. The examination was mainly conducted while standing. If arch height and hindfoot valgus could be corrected while standing on one's toes, the flatfoot was termed flexible (Great toe extension test). Those who could not recover their arch when standing on their toes were labeled as rigid pes planus and were barred to participate in this investigation.

The participants' recruitment process is shown in Figure 1. The inclusion criteria were as follows: (1) bilateral flexible flatfeet; (2) normal gait pattern without any mobility aids; (3) normal BMI. The participants were excluded if they had: history of neurological disorders (eg, cerebral palsy), neuromuscular diseases, genetic conditions or syndromes affecting gait or posture (eg, Down's

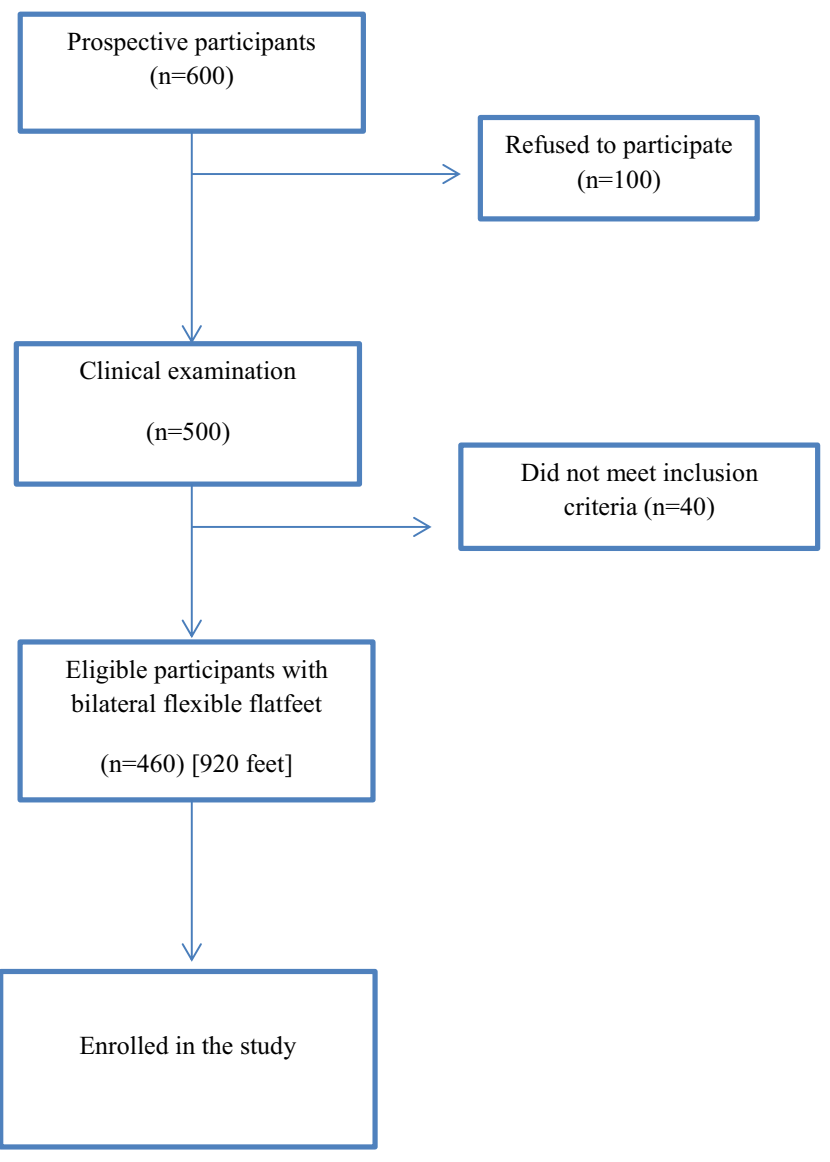

Figure I Enrollment flow chart of the study participants.

syndrome), congenital abnormalities of the foot (eg, tarsal coalition, vertical talus), structural lower extremity deformities, foot shape or size asymmetry between right and left sides, Joint rheumatic pathology, history of foot or ankle fractures or major trauma affecting ankle joint stability, history of major surgeries in the lower limbs, peripheral vascular disorders.

This study was carried out according to Declaration of Helsinki. The study was approved by the Human Research Ethics Committee of Ministry of health and prevention, UAE. The objectives and procedures of the study were explained in detail for the participants and their legal guardians before starting the study. Regarding the participants under 16 years old, signed written informed consent forms were obtained from the participants themselves (children's consent assent) and by their legal guardians (parental consent forms) prior to the commencement of the study protocol; signed informed consent forms were collected from the participants aged over 16 years old. This study was reported following the STROBE checklist. $^{41}$ 


\section{Measurement}

\section{Demographic Data}

Every participant was assigned an identification (ID) number. Each participant provided his/her age (year), gender, height $(\mathrm{cm})$, weight $(\mathrm{kg})$, and BMI (Table 1). A calibrated altimeter was used to determine the height (SECA 217 stadiometer, SECA, Hamburg Deutschland). Weight was measured using calibrated digital scales (Pegasus, Salter Brecknell DCSB, Avery Weigh-Tronix, Fairmont, USA) with subjects standing in bare feet and wearing just the essential necessities. The standard Quetelet index (body weight divided by height squared; $\mathrm{kg} / \mathrm{m} 2$ ) was used to compute BMI.

Among the factors affecting foot posture, BMI has still deemed the most controversial one in the available literature with some studies supporting its association with pronated feet, ${ }^{42,43}$ while, others are refuting this relationship. ${ }^{6}$ BMI was considered a confounding variable in the current study because soft tissue morphology is influenced by excessive weight (in overweight and obese subjects). ${ }^{44}$ Therefore, the obtained results of static foot posture assessment using either FPI-6 or any footprint indices will be absolutely affected. Furthermore, it has been reported that excessive BMI is associated with excessive abnormal loading of the planter zones of the adolescent foot, which may consequently lead to development of musculoskeletal disorders and serious postural abnormalities later in life. ${ }^{8,9,43}$ So, only participants with normal BMI were admitted, as a strategy to tightly control the confounders. ${ }^{45,46}$ BMI-based classification system for childhood obesity proposed by the International Obesity Task Force (IOTF) was followed in this study.

\section{Clinical Measurements of Static Foot Posture}

Clinical assessments were carried out by an impartial examiner who was not aware of the participants' identities

Table I Demographic Characteristics of the Participants $(n=460)$

\begin{tabular}{|l|l|}
\hline Characteristics & Participants (I 2-18 Years) \\
\hline Age $(\mathrm{y})$ & $15.23(0.84)$ \\
Height $(\mathrm{M})$ & $1.65(0.07)$ \\
Weight $(\mathrm{Kg})$ & $49.22(4.02)$ \\
BMI $\left(\mathrm{Kg} / \mathrm{m}^{2}\right)$ & $17.75(0.76)$ \\
Male & $300(49 \%)$ \\
Female & $312(5 \mathrm{I} \%)$ \\
\hline
\end{tabular}

Note: Continuous data are presented as mean (standard deviation) while categorical data are presented as number (\%).

Abbreviations: Y, year; M, Meter; Kg, Kilogram. or the study objectives. The examiner was an experienced physiotherapist with vast experience in evaluating static foot posture using FPI-6 and footprint measures such as CA. Before taking the measurement, the examiner went through a four-hour training session on how to use the FPI-6 and CA to evaluate static foot posture. Two research assistants [physiotherapy practitioners] were in charge of arranging clinical measurements for participants and organizing the measures processes.

For the measurement of CA, a static footprint was obtained. Each participant was asked to step onto a felt pad impregnated with a water-soluble ink with both feet and press firmly over the ink pad; then, the participant was asked to step forward placing his right foot onto a graph sheet with the participant's ID number, then his/her left foot is placed onto the graph sheet. The participant was asked to stand stable in a relaxed bipedal stance position with equal weight on both feet and looking forward for two seconds, and then step forward to clear the graph sheet.

If the footprint was not clear because of the inadequate amount of ink, the footprint was discarded and the procedure was repeated to obtain a good footprint. A transparent adhesive material was then placed over each foot print to keep it intact and the footprint sheets were collected in the footprint collection box.

CA was calculated using a marker pen, ruler and protractor marked at one degree interval. CA was obtained by calculating the angle between the medial tangential line (AB line in Figure 2) that connects the medial edges of the first metatarsal head and the heel, and the second line (AC line in Figure 2) that connects the first metatarsal head and the acme of the MLA concavity. ${ }^{15,16,18,23}$ Clarke's angle is considered as "normal" (CA $42^{\circ}-54^{\circ}$ ), "mild flat foot" (CA $35^{\circ}-41^{\circ}$ ), "moderate flatfoot" (CA $\left.30^{\circ}-34.9^{\circ}\right)$, "severe flat foot" (CA < $\left.30^{\circ}\right)$ and "high arched foot" $\left(\mathrm{CA}>54^{\circ}\right) .8,15,47$

The scoring system, methodology, reference values, and assessment technique given by Redmond for FPI- $6^{48}$

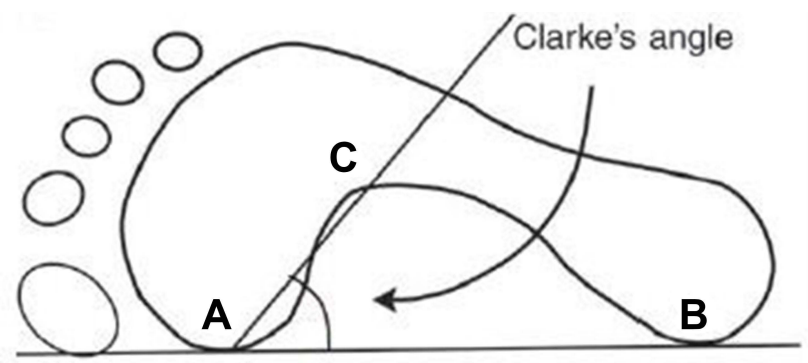

Figure 2 Clarke's angle. 
were used in this investigation. Each participant was instructed to stand in a comfortable stance with double limb support (same weight on both feet), arms by side, and eyes straight ahead. The participant was asked not to twist in an attempt to watch the evaluator, since this would impair foot posture and result in a fake scoring of FPI$6 .^{31}$ To eliminate rater bias, static foot posture was evaluated using FPI-6 the next day following footprint collection.

Foot posture was quantified utilizing FPI-6 criteria. This type of evaluation has six anatomical criteria, which are each graded from 0 (neutral) to +1 or +2 (pronated) and 1 or 2 (supinated): (1) Talar head palpation, in which the talus head is palpated in the front of the ankle in medial and lateral aspects; (2) Supra and infra lateral malleolar curvature, which is seen at the back of the ankle region (3) Calcaneal frontal plane position, where the orientation of the calcaneal tendon on the supporting surface is used as a reference. (4) Talonavicular joint bulging (5) Medial longitudinal arch height and congruence (6) Forefoot abduction or adduction $^{29,48}$ as shown in Figure 3.

A total score of 0 to +5 is defined as normal, +6 to +9 as pronated, +10 to +12 as strongly pronated, -1 to -4 as supinated, and -5 to -12 as strongly supinated foot. The ultimate score is a number between -12 and +12 (highly supinated and highly pronated, respectively). Inter-rater reliability had already been studied and confirmed for $\mathrm{CA}^{49}$ and FPI-6. ${ }^{23,31}$ To explore intra-rater reliability, all CA and FPI-6
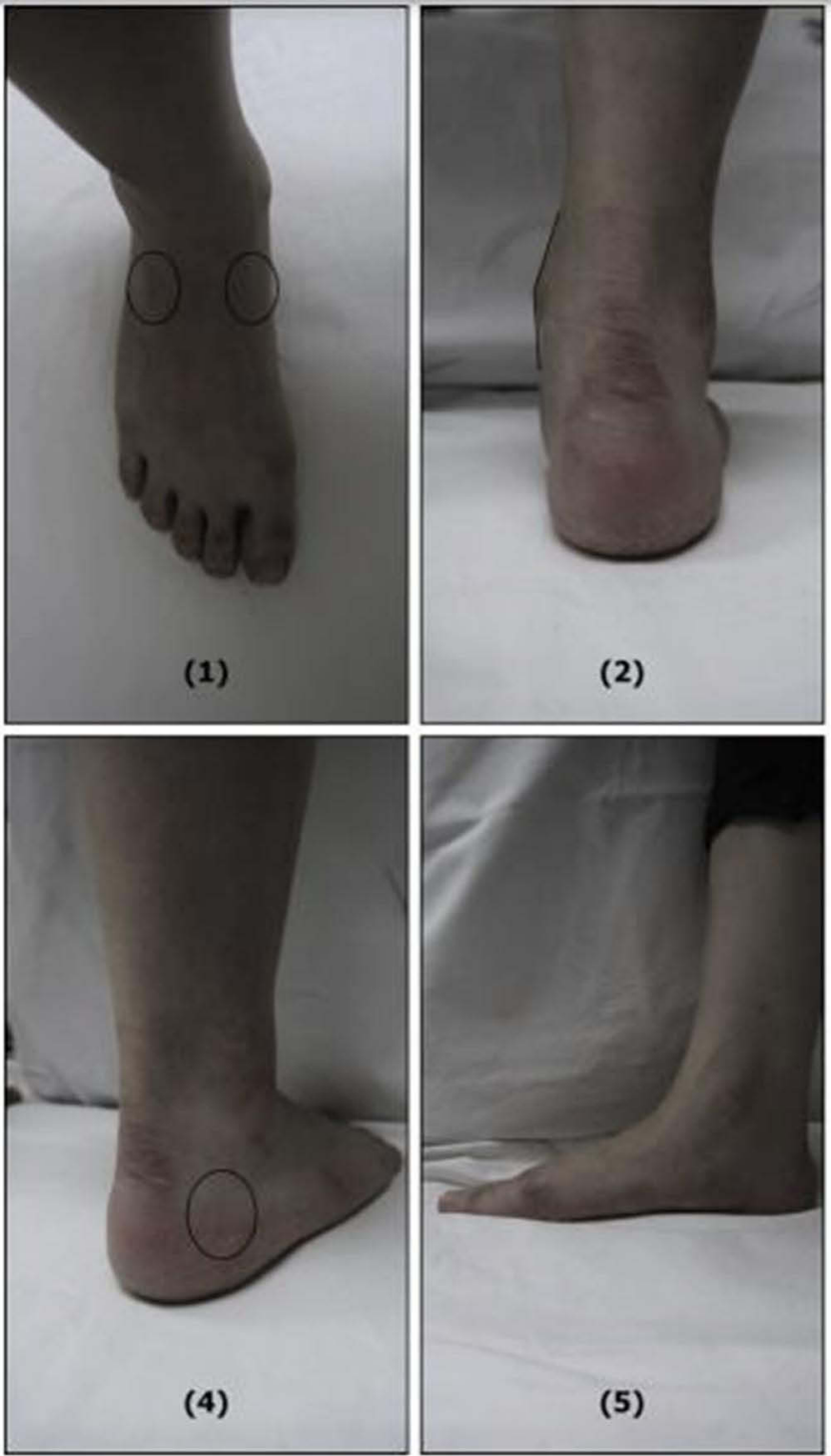
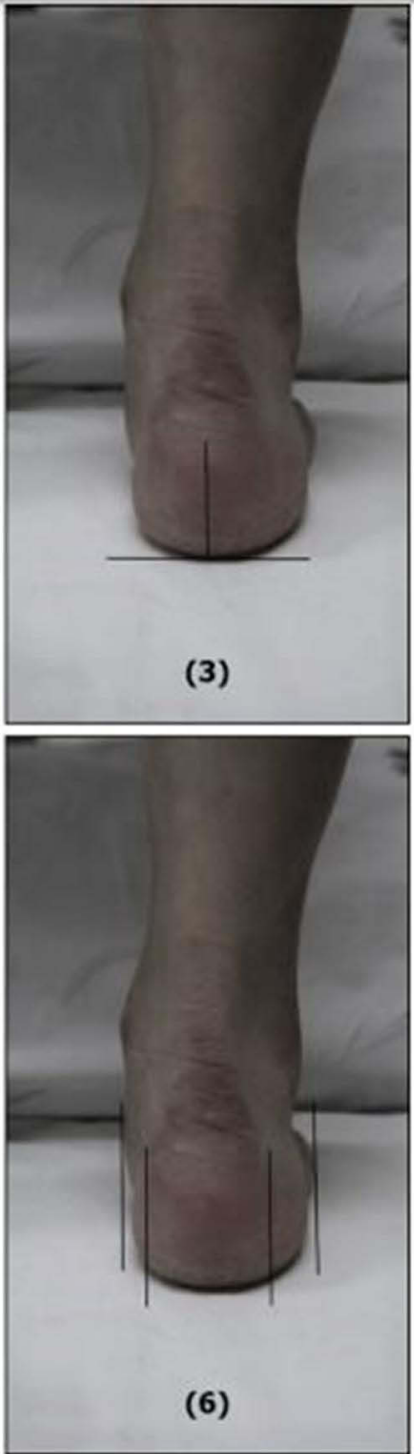

Figure 3 Six items of the foot posture index-6 (FPI-6). 
measurements were replicated by the same examiner one week apart.

\section{Radiographic Measurements}

Radiographic assessment is an extremely reliable criterion standard measure for the evaluation of foot alignment in the position of static weight bearings. ${ }^{32,50}$

The feet of the participants were radiographed from a comfortable weight-bearing standing position with equal weight on both feet. The participant stood on a wooden platform, knees extended and heels and toes at the same level. The X-ray film pack was positioned between the feet vertically. The X-ray tube was parallel to the ground, with the central beam focused at the midfoot. The distance between the tube and the film was $105 \mathrm{~cm}$, and the $\mathrm{X}$-ray was set to $2.8 \mathrm{~mA}$ at $45 \mathrm{kV}$.

Participants' lateral weight-bearing radiographs were seen on the Philips Easy Vision viewer (Philips Healthcare, Guildford, UK), radiographic measurements were performed with the Philips Easy Viewer software tools. The films were anonymized by eliminating any identifying information from them. There are several angular radiographic measures used for diagnosing flatfoot such as T1MA, Calcaneal Pitch angle (CP), Lateral Talocalcaneal angle (LTC). ${ }^{51}$ T1MA was chosen to represent static foot posture based upon its reliability, ease of measurement, and degree of resemblance to static foot posture. $^{52,53}$ Measurement of T1MA was performed by an experienced radiologist who was not aware of the study purposes.

T1MA is the angle formed by the talus and the first metatarsal bone's longitudinal axes. The midline axis of the talus bone aligns with the midline axis of the first metatarsal bone in the normal foot; however, in the

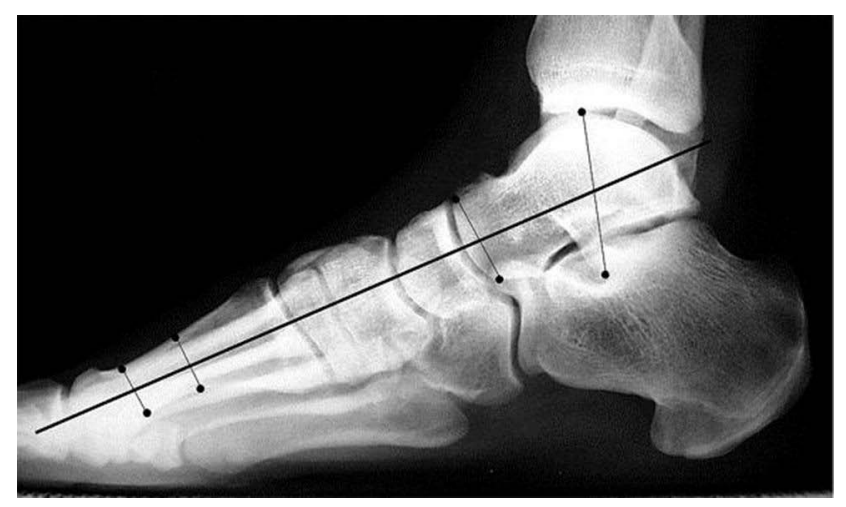

Figure 4 Normal talus first metatarsal angle (axis of talus coincides with axis of first metatarsal bone). pronated foot, the axis of the talus bone does not coincide with the midline axis of the first metatarsal bone (Figure 4). Normally, T1MA ranges from $-4^{\circ}$ to $+4^{\circ}$; T1MA greater than $+4^{\circ}$ (convex upward) suggests pes cavus; T1MA less than $4^{\circ}$ (convex downward) confirms flatfoot. $^{52}$

\section{Statistical Analysis}

The continuous data were presented as mean (standard deviation $[\mathrm{SD}]$ ), while the categorical data were presented as an absolute value (n) and percentage (\%).

To test the validity of CA and FPI-6 in detecting flatfoot, each participant's left and right feet were treated separately [920 feet]. Using the Intraclass correlation coefficients $(\mathrm{ICC})$ model $(3,1)$, the intra-rater reliability of FPI6 and CA was investigated. Because BMI was designated a confounding variable in this trial, only those with a normal BMI were accepted to the trial protocol, as an attempt to keep any potentially confounding variables under tight control. ${ }^{45}$

The sensitivity and specificity for each measure were computed, and the data were shown on a receiver operating characteristic (ROC) curve to examine the validity of CA and FPI-6 using T1MA as a criterion-standard measure. The area under the ROC curve (AUC) was computed as a performance indicator for the test.

The ROC curve is a widely used tool for evaluating the diagnostic accuracy of tests, and it fits very well with continuous data. The results from the FPI-6 and $\mathrm{CA}$ were displayed in $\mathrm{XY}$ directions, with the $\mathrm{X}$-axis representing the false-positive rate (1 specificity) and the Y-axis representing the true positive rate (TPR, sensitivity). ${ }^{20}$ The AUC value ranges from 0.5 to 1 , with high accuracy (AUC $>0.9$ ), moderate accuracy $(\mathrm{AUC}=0.7-0.9)$, poor accuracy $(\mathrm{AUC}=0.5-0.7)$, and a result of chance (AUC 0.5). ${ }^{20}$

The likelihood ratios (LRs) and positive and negative predictive values $(\mathrm{PV}+, \mathrm{PV})$ were determined. The Fagan nomogram was utilized to evaluate the post-test probability of flatfoot using FPI-6 and CA.

The sample size was computed with a confidence level of $95 \%$ [at $5 \%$ type 1 error ( $\mathrm{p} 0.05$ )], a power level of $80 \%$, and precision of $5 \%$. The current study required a minimum sample size of 400 individuals. A total of 460 people were recruited for the study, with a dropout rate of $10 \%$ to $15 \%$ expected. All statistical analyses were carried out with IBM SPSS statistics for Windows, version 23. (Armonk, NY: IBM Corp). The statistical significance level was selected at $\mathrm{P}<0.05$. 
Table 2 Descriptive Statistics for the Studied Clinical and Radiographic Measures ( $n=920$ Feet)

\begin{tabular}{|l|l|l|l|}
\hline Parameters & $\begin{array}{l}\text { FPI-6 } \\
\mathbf{n = 9 2 0}\end{array}$ & $\begin{array}{l}\text { CA }\left(^{\mathbf{0}} \text { ) }\right. \\
\mathbf{n = 9 2 0}\end{array}$ & $\begin{array}{l}\text { TIMA( } \\
\mathbf{n = 9 2 0}\end{array}$ \\
\hline Mean & 9.55 & 36.71 & -7.09 \\
SD & 0.74 & 3.20 & 4.03 \\
Minimum & 6.0 & 10.0 & -19.0 \\
Maximum & 10.0 & 42.0 & 4.0 \\
\hline
\end{tabular}

Abbreviations: FPI-6, foot posture index-6; CA, Clarke's angle; TIMA, talus first metatarsal angle; SD, standard deviation; ${ }^{0}$, degree.

\section{Results}

In this study, there were no missing data. Table 2 shows descriptive statistics for the clinical and radiological measures that were investigated. As indicated in Table 3, FPI-6 had a great intra-rater reliability $[\mathrm{ICC}=0.96$ ] with a $95 \%$ confidence interval $(95 \% \mathrm{CI})$ of $0.95-0.98$, whereas CA had a greater intra-rater reliability $[\mathrm{ICC}=0.99 ; 95 \% \mathrm{CI}$ (0.997-0.998)] with $\mathrm{P}<0.001$ (3).

The sensitivity of FPI-6 and CA was $84 \%$ and $98 \%$, respectively, whereas the specificity of FPI- 6 and CA was $80 \%$ and $97 \%$, respectively (Table 3 ). Cross-tabulation of FPI-6 and CA against T1MA is displayed in Table 4. ROC

Table 3 Statistical Parameters for the Studied Clinical Measures

\begin{tabular}{|c|c|c|}
\hline Parameters & $\begin{array}{l}\text { FPI-6 } \\
n=920\end{array}$ & $\begin{array}{l}C A \\
n=920\end{array}$ \\
\hline ICC $(95 \% \mathrm{Cl})$ & $\begin{array}{l}0.963 \\
(0.952-0.983)\end{array}$ & $\begin{array}{l}0.997 \\
(0.996-0.998)\end{array}$ \\
\hline Sensitivity & $84 \%$ & $98 \%$ \\
\hline Specificity & $80 \%$ & $99 \%$ \\
\hline AUC $(95 \% \mathrm{Cl})$ & $\begin{array}{l}0.80 \\
(0.77-0.85)\end{array}$ & $\begin{array}{l}0.98 \\
(0.95-1.00)\end{array}$ \\
\hline $\mathrm{PV}+$ & $64 \%$ & $98 \%$ \\
\hline PV- & $92 \%$ & $98.9 \%$ \\
\hline $\mathrm{LR}+$ & 4.21 & 97 \\
\hline LR- & 0.20 & 0.02 \\
\hline Pretest probability & $30 \%$ & $30 \%$ \\
\hline $\begin{array}{l}\text { Posttest probability }(\mathrm{LR}+) \\
{[95 \% \mathrm{Cl}]}\end{array}$ & $64 \%$ [59-66\%] & $98 \%[94-99 \%]$ \\
\hline $\begin{array}{l}\text { Posttest probability (LR-) } \\
{[95 \% \mathrm{Cl}]}\end{array}$ & $8 \%$ [3-9\%] & $1 \%[0-2 \%]$ \\
\hline
\end{tabular}

Abbreviations: FPI-6, foot posture index-6; CA, Clarke's angle; ICC, Intraclass correlation coefficient; $95 \% \mathrm{Cl}, 95 \%$ confidence interval; AUC, area under the ROC curve; $\mathrm{PV}+$, positive predictive value; $\mathrm{PV}-$, negative predictive value; $\mathrm{LR}+$, positive likelihood ratio; LR-, negative likelihood ratio.
Table 4 Cross-Tabulation for the Studied Clinical Measures Against TIMA ( $n=920$ Feet)

\begin{tabular}{|c|c|c|c|c|}
\hline & & \multicolumn{2}{|c|}{ TIMA } & \multirow[t]{2}{*}{ Total } \\
\hline & & $\mathbf{P}$ & $\mathbf{N}$ & \\
\hline \multirow[t]{2}{*}{ CA } & $P$ & $27 \mathrm{I}(29.45 \%)$ & 7 (0.76\%) & $350(28.60 \%)$ \\
\hline & $\mathrm{N}$ & 5 (0.54\%) & 637 (69.24\%) & 874 (7I. $40 \%)$ \\
\hline \multicolumn{2}{|l|}{ Total } & $276(30 \%)$ & $644(70 \%)$ & 920 (100\%) \\
\hline \multirow[t]{2}{*}{ FPI-6 } & $P$ & $232(52.22 \%)$ & 128 (13.91\%) & 459 (37.5\%) \\
\hline & $\mathrm{N}$ & 44 (4.78\%) & 516 (56.86\%) & 765 (62.5\%) \\
\hline \multicolumn{2}{|l|}{ Total } & 276 (30\%) & 644 (70\%) & 920 (100\%) \\
\hline
\end{tabular}

Note: Data are presented as absolute numbers (\%).

Abbreviations: TIMA, talus first metatarsal angle; CA, Clarke's angle; FPI-6, Foot posture index-6; P, positive result (flatfoot); N, negative result (normal).

curves for the clinical measures are shown in Figure 5 and the AUC was 0.98 with $95 \%$ CI of (0.95-1.00) for CA; and 0.81 with $95 \%$ CI of (0.77-0.85) for FPI-6 (Table 3).

The PV+ values for CA and FPI- 6 were $98 \%$ and $64 \%$, respectively, and the PV- values for CA and FPI-6 were $98.9 \%$ and $92 \%$, respectively (Table 3 ). Likelihood ratios (LRs) were computed and the post-test probabilities were displayed on Fagan nomogram. LR+ values for CA and FPI-6 were 97 and 4.21, respectively, and the LR- values for CA and FPI-6 were 0.02, and 0.20, respectively.

The pre-test probability was determined to be $30 \%$ in the current study. LR+ showed a post-test probability of $98 \%$ for CA and $64 \%$ for FPI-6, while LR- provided a post-test probability of $1 \%$ for CA and $8 \%$ for FPI-6 (Table 3 ) as plotted on Fagan nomogram (Figure 6).

\section{Discussion}

This study aimed to investigate and compare validity and diagnostic accuracy of FPI-6 and CA in identifying flexible flatfoot in adolescents aged 12-18 years utilizing radiographic findings as a reference standard measure.

\section{Reliability of FPI-6 and CA}

Inter-rater reliability had already been investigated and reported for $\mathrm{CA}^{49}$ and FPI-6, ${ }^{23,31}$ thus this study did not examine it. FPI-6 demonstrated outstanding intra-rater reliability ( $\mathrm{ICC}=0.96,95 \% \mathrm{CI}$ ) according to the findings of the current investigation $(0.95-0.98)$. This contradicted the findings of a prior study, ${ }^{34}$ which indicated moderate FPI test-retest reliability $(\mathrm{ICC}=0.61)$. This disparity could be explained by the study's use of an older version of FPI (FPI-8) against the current study's use of the modified version of FPI (FPI-6). Furthermore, their sample size 


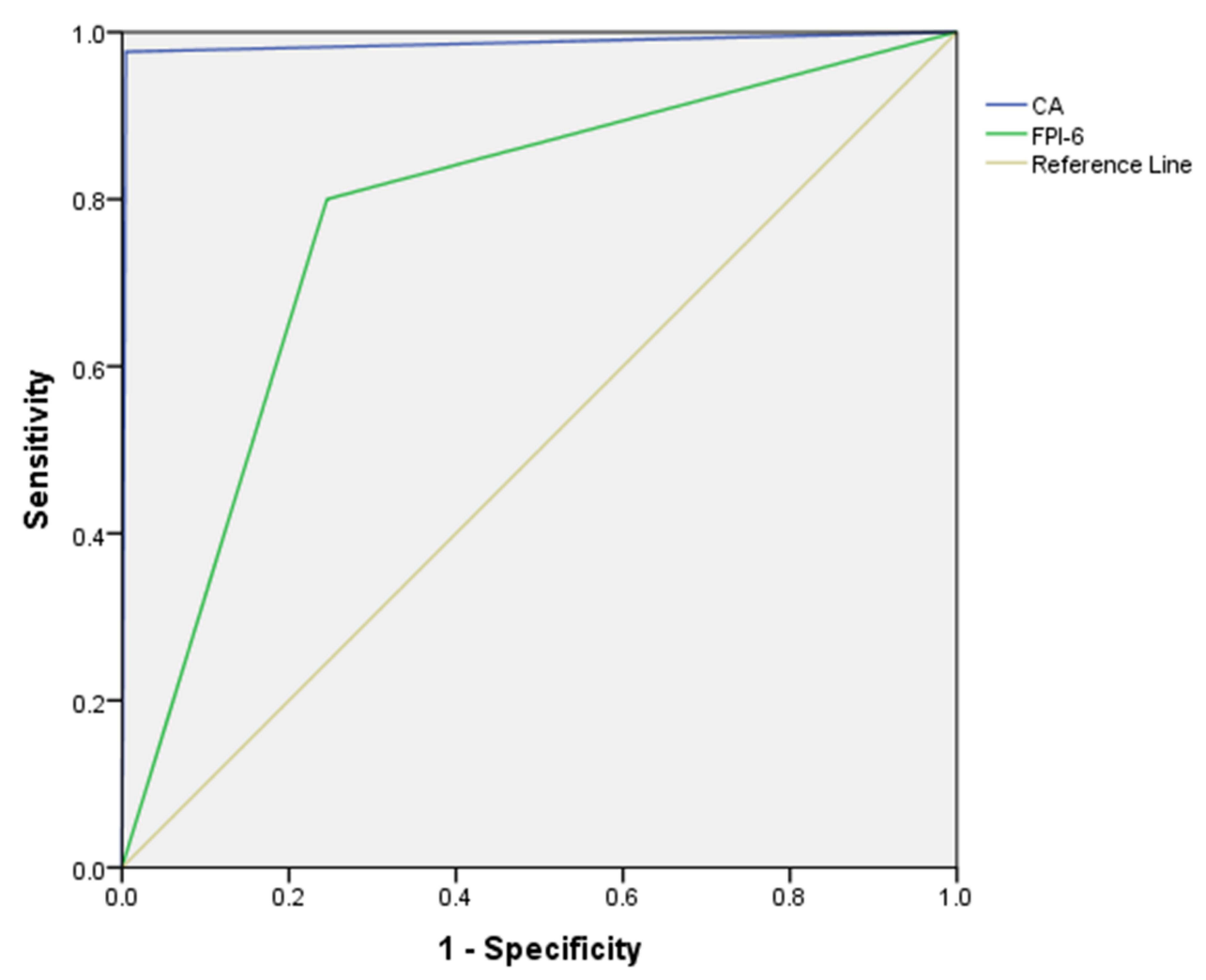

Figure 5 ROC curve for FPI-6 and Clark's angle.

$(n=95)$, inclusion criteria, and participants' ages (62-94 years) were not the same as those in the current study. Furthermore, the BMI was not controlled in this study because the mean BMI in their group was $26.8 \mathrm{~kg} / \mathrm{m} 2$, which is deemed overweight according to WHO criteria. ${ }^{54}$ As a result, because soft tissue morphology is thought to be influenced by BMI, this could impact the outcomes of FPI-based foot posture examination especially in subjects with higher BMI (overweight or obese).

Evans et $\mathrm{al}^{55}$ evaluated the inter-rater and intra-rater reliability of FPI-6 in adolescents aged 7-15 years and found high intra-rater reliability of FPI-6 (ICC > 0.92).

The intra-rater reliability of Clarke's angle (CA) was excellent $[\mathrm{ICC}=0.99 ; 95 \% \mathrm{CI}$ of $0.997-0.998]$. These findings are in line with prior research; $;^{26,56,57}$ which found that CA had high intra-rater reliability (ICC > 0.9). Based upon the current study findings, both CA and FPI-6 exhibit great intra-rater reliability.

\section{Sensitivity and Specificity of FPI-6 and CA}

CA is more sensitive (98\%) and specific (99\%) than FPI-6, according to the findings of this investigation. This could be due to the fact that FPI-6 is an observational scoring system with some subjectivity, which could lead to measurement inaccuracies. To the authors' knowledge, this is the first study to evaluate and compare the validity and diagnostic accuracy of CA and FPI-6 against the same criterion standard measure in the same population sample under the same research circumstances.

Pita-Fernandez et $\mathrm{al}^{20}$ evaluated the validity of three footprint measures (CA, Chippaux-Smirak index, and Staheli index) for diagnosing flat foot in senior persons aged 40 to 64 years. They found that CA had a higher sensitivity (83.71\%) and specificity (90.7\%) than the other studied measures. Furthermore, a cross-sectional study published recently ${ }^{36}$ evaluated the validity of $\mathrm{CA}$ and arch index (AI) in identifying flatfoot in children aged 310 years. They reported that CA had a high sensitivity (90\%) and specificity (90\%). They did not, however, include FPI-6 in their investigation, and their sample size was quite small $(\mathrm{n}=84)$ in comparison to with the current investigation.

\section{ROC Curve for FPI-6 and CA}

The ROC curve is a widely used method for assessing the diagnostic accuracy of tests by measuring the area under the ROC curve (AUC) ${ }^{15,20} \mathrm{CA}$ demonstrated a higher AUC value (0.98) with $95 \%$ CI $(0.95-1.00)$ than FPI-6 

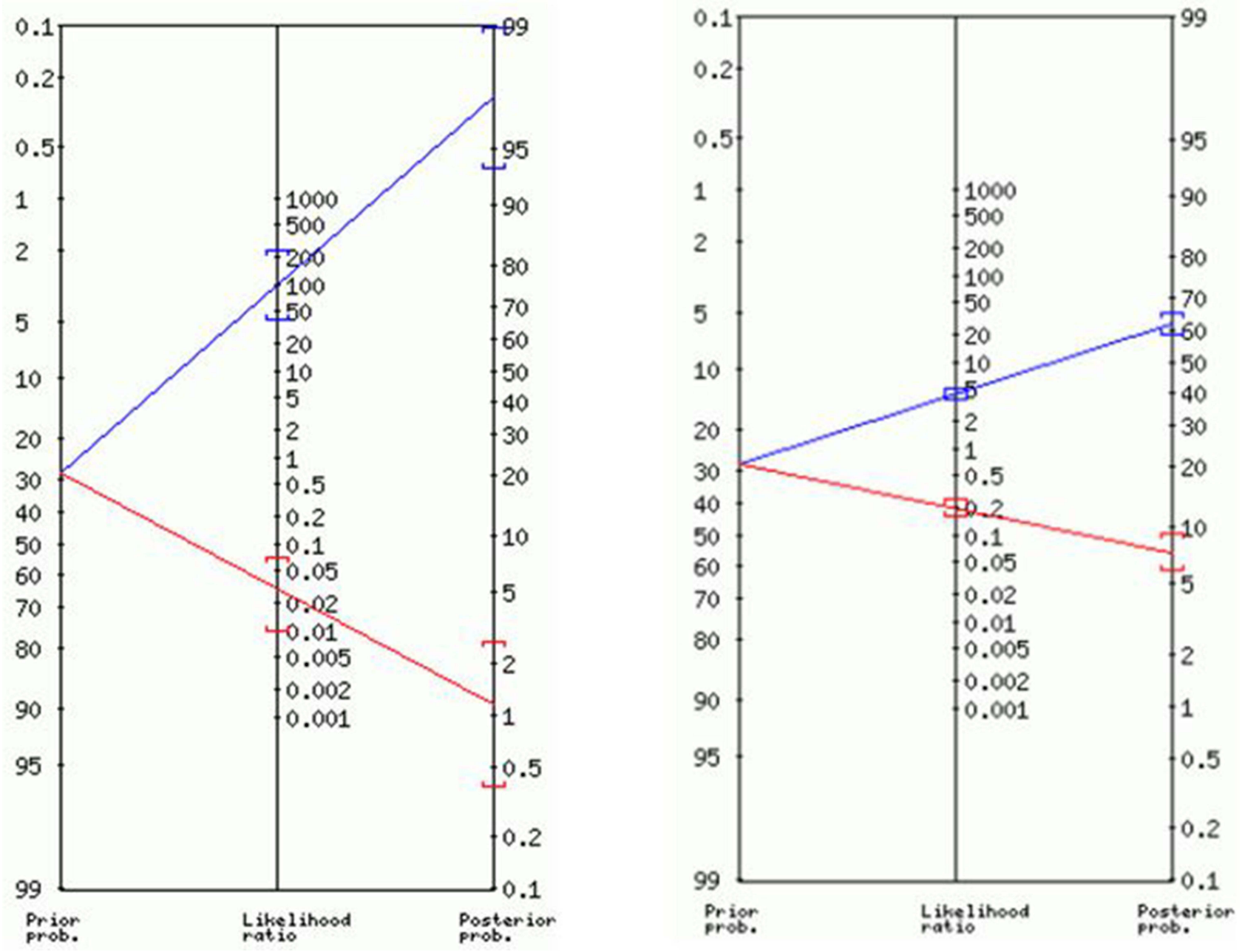

Figure 6 Fagan nomogram for CA (on the left side) and FPI-6 (right side) in adolescents aged I2-18 years.

$[\mathrm{AUC}=0.81 ; 95 \% \mathrm{CI}(0.77-0.85)]$, indicating a higher diagnostic accuracy of CA than FPI-6.

Pita-Fernández et $\mathrm{al}^{20}$ found that $\mathrm{CA}$ had the highest diagnostic accuracy (AUC $=0.93$ ) in adults over the age of 40 years. Similarly, Hameed et $\mathrm{al}^{36}$ demonstrated highest diagnostic accuracy of CA $(\mathrm{AUC}=0.95)$ in children aged 3 10 years diagnosed with flatfeet. Chen et al, ${ }^{15}$ on the other hand, reported moderate diagnostic accuracy of CA (AUC= $0.90)$ in healthy preschool children aged 3-6 years. However, comparing results to those of the current study is problematic due to differences in sample characteristics (age, sample size), as flatfeet is the typical foot posture at this age (3-6 years) due to immature osseous structures, ligamentous laxity, and high adipose tissue thickness. ${ }^{15}$ Furthermore, the methodology followed was different as they did not include FPI-6 in the studied clinical measures and they used clinical diagnosis as a criterion standard measure. In contrast, the current study used radiographic measure as a criterion standard measure which is more objective than clinical diagnosis.

\section{Predictive Values for FPI-6 and CA}

Once the test findings are known, predictive values (PVS) show the patient's chances of having or not having a condition. PVS are divided into two categories: positive $(\mathrm{PV}+)$ and negative (PV). PVs are identified as an efficient indicator of diagnostic accuracy. ${ }^{58}$

CA had a higher PV+ (98\%) than FPI-6 (64\%) and a higher PV (98.9\%) than FPI-6 (92\%) in the current investigation, showing that CA has a higher accuracy in diagnosis of flatfoot than FPI-6. In other words, a person who receives a positive result (abnormal CA) during a static foot posture evaluation with $\mathrm{CA}$ has a probability of $98 \%$ of having 
abnormal static foot posture (flat foot). An individual with a negative result (normal CA) has a $98.9 \%$ chance of having normal static foot posture.

In healthy preschool children aged 3-6 years, Chen et $\mathrm{al}^{15}$ reported that $\mathrm{PV}+$ and $\mathrm{PV}-$ for $\mathrm{CA}$ were $84 \%$ and $82 \%$, respectively, in healthy preschool children aged 3-6 years. These results are inconsistent with our results that might be attributed to the differences in the sample characteristics such as young age (3-6 years old), larger sample size than the current study (1319 participants), and unclear inclusion criteria for the participants (preschool children), which makes it difficult to compare with the current study results.

\section{Likelihood Ratios for FPI-6 and CA}

For a more in-depth look at diagnostic accuracy, likelihood ratios (LRs) for CA and FPI-6 were determined. LRs are a measure of a test's efficiency and diagnostic accuracy since they combine the sensitivity and specificity of the test to estimate the disease probability. ${ }^{59} \mathrm{LR}+(97)$ was substantially greater in CA than in FPI-6 (4.21). This suggests that someone with abnormal foot posture is 97 considerably more probable than someone with normal static foot posture to get a positive CA test result. In addition, CA had a lower LR (0.02) than FPI-6 (0.20). CA has higher diagnostic accuracy than FPI-6, according to the results of the sample.

The expected probability of the disease before obtaining the test result is defined as the pre-test probability (prior probability). In the current investigation, the pre-test probability was determined to be $30 \%$. The patient's probability of having the disease after the test findings are obtained is defined as the post-test probability (posterior probability). The post-test probability fluctuates as LRs are varied. ${ }^{59}$

The Fagan nomogram was used to estimate the probability of flatfoot based on the LRs in this investigation. The pretest probability of flatfoot was $30 \%$ in the present study. LR+ yielded a post-test probability of $98 \%$ for CA and $64 \%$ for FPI-6, whereas LR- led in a post-test probability of $1 \%$ for CA and $8 \%$ for FPI-6. Based upon the current investigation, CA has a superior diagnostic accuracy than FPI-6 in identifying flatfoot in adolescents aged 12 to 18 with normal BMI.

\section{Clinical Significance}

Flatfoot is linked to the development of a variety of musculoskeletal problems. Finding the best reliable and valid clinical assessment measure of static foot position is therefore beneficial for both clinicians and researchers, as it refines and enriches clinical practice. This study provides evidence for the validity, predictive capacity, and diagnostic accuracy of the CA and FPI-6 in teenagers. This evidence will increase physicians' confidence in the diagnosis and identification of static foot posture disorder without such additional radiographic examination and ionizing radiation exposure. As a result, there will be a beneficial impact on refining and enhancing the evidence-based clinical practice.

Furthermore, after evaluating the validity of CA against FPI- 6 in the same sample against the same criterion standard measure, the current study provides scientific proof to settle the ongoing argument concerning its validity and diagnostic accuracy.

\section{Strength Points}

- This is the first study to investigate and compare the validity and diagnostic accuracy of FPI-6 and Clark's angle against one criterion standard measure (radiographic findings) in the same sample under the same circumstances.

- Targeting foot posture in adolescents helps researchers and clinicians to understand and track the developmental trajectory of the foot through this stage of life, as foot posture changes with growth and developmental maturation from childhood through adolescence to adulthood.

- Investigating validity and diagnostic accuracy of FPI6 and CA as commonly used clinical assessment measures of foot posture, supports evidence-based practice as this knowledge facilitates the proper diagnosis of flatfoot in adolescents based upon choosing the most valid and accurate clinical measure leading to proper decision making regarding intervention.

- The present study focused on narrow age range (adolescents aged 12-18 years) which improves the external validity of the results obtained compared with the majority of the previous studies that studied a wider age range of the participants.

\section{Limitations}

The convenience sampling used in this study limits the generalizability of the findings. Furthermore, because the current study only addressed the validity and diagnostic accuracy of CA and FPI-6 in adolescents with a normal BMI, more research is needed to investigate the validity and diagnostic accuracy of these clinical measures in diverse groups with varying BMI values (underweight, overweight, obese). 


\section{Conclusion}

CA and FPI-6 are both valid and diagnostically accurate clinical tools for identifying flexible flatfoot with a superior performance of CA in adolescents aged 12 to 18 years with a normal BMI. Based upon the present study, CA can be utilized as a screening method for flatfoot in adolescents with normal BMI.

\section{Data Sharing Statement}

All relevant data are presented within the manuscript.

\section{Author Contributions}

All authors made a significant contribution to the work reported, whether that is in the conception, study design, execution, acquisition of data, analysis and interpretation, or in all these areas; took part in drafting, revising or critically reviewing the article; gave final approval of the version to be published; have agreed on the journal to which the article has been submitted; and agree to be accountable for all aspects of the work.

\section{Disclosure}

The authors declare that they have no conflicts of interest.

\section{References}

1. Banwell HA, Paris ME, Mackintosh S, Williams CM. Paediatric flexible flat foot: how are we measuring it and are we getting it right? A systematic review. J Foot Ankle Res. 2018;11(1). doi:10.1186/s13047-018-0264-3

2. Boryczka-Trefler A, Kalinowska M, Szczerbik E, Stępowska J, Łukaszewska A, Syczewska M. How to define pediatric flatfoot: comparison of 2 methods: foot posture in static and dynamic conditions in children 5 to 9 years old. Foot Ankle Spec. 2021;193864002199134. doi:10.1177/1938640021991345

3. Carrasco AC, Silva MF, Guenka LC, Silva CT, Moura FA, Cardoso JR. Non-radiographic validity and reliability measures for assessing foot types: a systematic review. Foot Ankle Surg. 2021. Elsevier Ltd. doi:10.1016/j.fas.2020.11.011

4. de Carvalho BKG, Penha PJ, Penha NLJ, Andrade RM, Ribeiro AP, João SMA. The influence of gender and body mass index on the FPI-6 evaluated foot posture of 10- to 14-year-old school children in São Paulo, Brazil: a cross-sectional study. J Foot Ankle Res. 2017;10:1-7. doi:10.1186/s13047-016-0183-0

5. Müller S, Carlsohn A, Müller J, Baur H, Mayer F. Static and dynamic foot characteristics in children aged 1-13 years: a cross-sectional study. Gait Posture. 2012;35:389-394. doi:10.1016/j. gaitpost.2011.10.357

6. Gonçalves de Carvalho BK, Penha PJ, Ramos NLJP, Andrade RM, Ribeiro AP, João SMA. Age, sex, body mass index, and laterality in the foot posture of adolescents: a cross sectional study. J Manipulative Physiol Ther. 2020;43:744-752. doi:10.1016/j.jmpt.2018.11.035

7. Kothari A, Dixon PC, Stebbins J, Zavatsky AB, Theologis T. Are flexible flat feet associated with proximal joint problems in children? Gait Posture. 2016;45:204-210. doi:10.1016/j.gaitpost.2016.02.008
8. Szczepanowska-Wolowiec B, Sztandera P, Kotela I, Zak M. Feet deformities and their close association with postural stability deficits in children aged 10-15 years. BMC Musculoskelet Disord. 2019;20:537. doi:10.1186/s12891-019-2923-3

9. Szczepanowska-Wolowiec B, Sztandera P, Kotela I, Zak M. Vulnerability of the foot's morphological structure to deformities caused by foot loading paradigm in school-aged children: a cross-sectional study. Sci Rep. 2021;11:2749. doi:10.1038/s41598-021-82475-y

10. Levy JC, Mizel MS, Wilson LS, et al. Incidence of foot and ankle injuries in west point cadets with pes planus compared to the general cadet population. Foot Ankle Int. 2006;27:1060-1064. doi:10.1177/ 107110070602701211

11. Tenenbaum S, Hershkovich O, Gordon B, et al. Flexible pes planus in adolescents: body mass index, body height, and gender - an epidemiological study. Foot Ankle Int. 2013;34:811-817. doi:10.1177/ 1071100712472327

12. Varga M, Price C, Morrison SC. Three-dimensional foot shape analysis in children: a pilot analysis using three-dimensional shape descriptors. J Foot Ankle Res. 2020;13:6. doi:10.1186/s13047-0200373-7

13. Stavlas P, Grivas TB, Michas C, Vasiliadis E, Polyzois V. The evolution of foot morphology in children between 6 and 17 years of age: a cross-sectional study based on footprints in a Mediterranean population. J Foot Ankle Surg. 2005;44:424-428. doi:10.1053/j. jfas.2005.07.023

14. Hegazy FA, Aboelnasr EA, Salem Y, Zaghloul AA. Validity and diagnostic accuracy of foot posture index-6 using radiographic findings as the gold standard to determine paediatric flexible flatfoot between ages of 6-18 years: a cross-sectional study. Musculoskelet Sci Pract. 2020;46:102107. doi:10.1016/j.msksp.2020.102107

15. Chen KC, Yeh CJ, Kuo JF, Hsieh CL, Yang SF, Wang CH. Footprint analysis of flatfoot in preschool-aged children. Eur J Pediatr. 2011;170:611-617. doi:10.1007/s00431-010-1330-4

16. Razeghi M, Batt ME. Foot type classification: a critical review of current methods. Gait Posture. 2002;15(3):282-291. Elsevier Ireland Ltd. doi:10.1016/S0966-6362(01)00151-5

17. Aboelnasr EA, El-Talawy HA, Abdelazim FH, Hegazy FA. Sensitivity and specificity of normalized truncated navicular height in assessment of static foot posture in children aged 6-12 years. Hong Kong Physiother J. 2019;39:15-23. doi:10.1142/ S1013702519500021

18. Billis E, Katsakiori E, Kapodistrias C, Kapreli E. Assessment of foot posture: correlation between different clinical techniques. Foot. 2007;17:65-72. doi:10.1016/j.foot.2006.09.005

19. Gonzalez-Martin C, Pita-Fernandez S, Seoane-Pillado T, LopezCalviño B, Pertega-Diaz S, Gil-Guillen V. Variability between Clarke's angle and Chippaux-Smirak index for the diagnosis of flat feet. Colomb Med. 2017;48:25-31. doi:10.25100/cm.v48i1.1947

20. Pita-Fernández S, González-Martín C, Seoane-Pillado T, LópezCalviño B, Pértega-Díaz S, Gil-Guillén V. Validity of footprint analysis to determine flatfoot using clinical diagnosis as the gold standard in a random sample aged 40 years and older. $J$ Epidemiol. 2015;25:148-154. doi:10.2188/jea.JE20140082

21. Drefus LC, Kedem P, Mangan SM, Scher DM, Hillstrom HJ. Reliability of the arch height index as a measure of foot structure in children. Pediatr Phys Ther. 2017;29:83-88. doi:10.1097/ PEP.0000000000000337

22. Hösl M, Böhm H, Oestreich C, et al. Self-perceived foot function and pain in children and adolescents with flexible flatfeet - relationship between dynamic pedobarography and the foot function index. Gait Posture. 2020;77:225-230. doi:10.1016/j.gaitpost.2020.01.014

23. Kerr CM, Stebbins J, Theologis T, Zavatsky AB. Static postural differences between neutral and flat feet in children with and without symptoms. Clin Biomech. 2015;30:314-317. doi:10.1016/j. clinbiomech.2015.02.007 
24. Kanatli U, Yetkin H, Cila E. Footprint and radiographic analysis of the feet. J Pediatr Orthop. 2001;21:225-228. doi:10.1097/01241398200103000-00018

25. Villarroya MA, Esquivel JM, Tomás C, Moreno LA, Buenafé A, Bueno G. Assessment of the medial longitudinal arch in children and adolescents with obesity: footprints and radiographic study. Eur J Pediatr. 2009;168:559-567. doi:10.1007/s00431-008-0789-8

26. Pauk J, Ihnatouski M, Najafi B. Assessing plantar pressure distribution in children with flatfoot arch: application of the Clarke angle. J Am Podiatr Med Assoc. 2014;104:622-632. doi:10.7547/87507315-104.6.622

27. Nikolaidou ME, Boudolos KD. A footprint-based approach for the rational classification of foot types in young schoolchildren. Foot. 2006;16:82-90. doi:10.1016/j.foot.2006.02.001

28. Pauk J, Szymul J. Differences in pediatric vertical ground reaction force between planovalgus and neutrally aligned feet. Acta Bioeng Biomech. 2014;16:95-101. doi:10.5277/abb140211

29. Gijon-Nogueron G, Martinez-Nova A, Alfageme-Garcia P, MontesAlguacil J, Evans AM. International normative data for paediatric foot posture assessment: a cross-sectional investigation. BMJ Open. 2019;9:e023341. doi:10.1136/bmjopen-2018-023341

30. Redmond AC, Crane YZ, Menz HB. Normative values for the foot posture index. J Foot Ankle Res. 2008;1:6. doi:10.1186/1757-1146$1-6$

31. Redmond AC, Crosbie J, Ouvrier RA. Development and validation of a novel rating system for scoring standing foot posture: the foot posture index. Clin Biomech. 2006;21:89-98. doi:10.1016/j. clinbiomech.2005.08.002

32. Kim KB, Lee JS, Jeong JO, Kwon NY, Jeong SM. Correlation of foot posture index with plantar pressure and radiographic measurements in pediatric flatfoot. Ann Rehabil Med Orig Artic Ann Rehabil Med. 2015;39:10-17. doi:10.5535/arm.2015.39.1.10

33. Morrison SC, McCarthy D, Mahaffey R. Associations between obesity and pediatric foot dimensions. J Am Podiatr Med Assoc. 2018;108:383-389. doi:10.7547/16-172

34. Menz HB, Munteanu SE. Validity of 3 clinical techniques for the measurement of static foot posture in older people. J Orthop Sports Phys Ther. 2006;36:179. doi:10.2519/jospt.2006.0201

35. Gijon-Nogueron G, Marchena-Rodriguez A, Montes-Alguacil J, Evans AM. Evaluation of the paediatric foot using footprints and foot posture index: a cross-sectional study. $J$ Paediatr Child Health. 2020;56:201-206. doi:10.1111/jpc.14558

36. Hameed N, Baseer N, Huma Z, Javed S, Raza T, Sadaf A. Anthropometric assessment of paediatric flat foot: a diagnostic accuracy study. J Ayub Med Coll Abbottabad. 2020;32:359-367.

37. Hegazy F, Aboelnasr E, Abdel-Aziem AA, Kim I-J. Validity and diagnostic accuracy of Clarke's angle in determining paediatric flexible flatfoot using radiographic findings as a criterion standard measure: a cross-sectional study. J Am Podiatr Med Assoc. 2021. doi:10.7547/20-133

38. Hollander K, Zech A, Rahlf AL, Orendurff MS, Stebbins J, Heidt $\mathrm{C}$. The relationship between static and dynamic foot posture and running biomechanics: a systematic review and meta-analysis. Gait Posture. 2019;72:109-122. Elsevier. doi:10.1016/j. gaitpost.2019.05.031

39. Buldt AK, Allan JJ, Landorf KB, Menz HB. The relationship between foot posture and plantar pressure during walking in adults: a systematic review. Gait Posture. 2018;62:56-67. doi:10.1016/j. gaitpost.2018.02.026

40. Gould N. Evaluation of hyperpronation and pes planus in adults. Clin Orthop Relat Res. 1983;181:37-45. doi:10.1097/00003086198312000-00007

41. Vandenbroucke JP, Von Elm E, Altman DG, et al. Strengthening the Reporting of Observational Studies in Epidemiology (STROBE): explanation and elaboration. PLoS Med. 2007;4:1628-1654. doi:10.1371/journal.pmed.0040297
42. Pita-Fernandez S, Gonzalez-Martin C, Aalonso-Tajes F, et al. Flat foot in a random population and its impact on quality of life and functionality. J Clin Diagnostic Res. 2017;11:22-27. doi:10.7860/ JCDR/2017/24362.9697

43. Szczepanowska-Wolowiec B, Sztandera P, Kotela I, Zak M. Body weight-dependent foot loads, assessed in terms of BMI and adiposity, in school-aged children: a cross sectional study. Sci Rep. 2020;10:12360. doi:10.1038/s41598-020-69420-1

44. Shree S, Revathi S, Thiyagarajan A, Kumar D. Does obesity cause flat foot? J Obes. 2018;2:1-5.

45. Pourhoseingholi MA, Baghestani AR, Vahedi M. How to control confounding effects by statistical analysis. Gastroenterol Hepatol Bed Bench. 2012;5:79-83. doi:10.22037/ghfbb.v5i2.246

46. Lu CY. Observational studies: a review of study designs, challenges and strategies to reduce confounding. Int $J$ Clin Pract. 2009;63:691-697. doi:10.1111/j.1742-1241.2009.02056.x

47. Forriol F, Pascual J. Footprint analysis between three and seventeen years of age. Foot Ankle Int. 1990;11:101-104. doi:10.1177/ 107110079001100208

48. Redmond A. The foot posture index: easy quantification of standing foot posture: six item version: FPI-6: user guide and manual. United Kingdom; 2005:1-19. Available from: https://scholar.google.com/ scholar? $\mathrm{q}=+$ The + foot + posture + index. + Easy + quantification + of + stand ing + foot + posture $\% 3 \mathrm{~A}+$ User + guide + and + manual. $+\& \mathrm{hl}=\mathrm{en} \approx \mathrm{sdt}=0 \%$ $2 \mathrm{C} 5 \approx \mathrm{ylo}=2005 \approx \mathrm{yhi}=2005$. Accessed September 10, 2021.

49. Tucker J, Moore M, Rooy J, Wright A, Rothschild C, Werk LN. Reliability of common lower extremity biomechanical measures of children with and without obesity. Pediatr Phys Ther. 2015;27:250-256. doi:10.1097/PEP.0000000000000152

50. Murley GS, Menz HB, Landorf KB. A protocol for classifying normal- and flat-arched foot posture for research studies using clinical and radiographic measurements. J Foot Ankle Res. 2009;2. doi:10.1186/1757-1146-2-22

51. Indino C, Villafañe JH, D'Ambrosi R, et al. Effectiveness of subtalar arthroereisis with endorthesis for pediatric flexible flat foot: a retrospective cross-sectional study with final follow up at skeletal maturity. Foot Ankle Surg. 2020;26:98-104. doi:10.1016/j.fas.2018.12.002

52. Waldt S, Eiber M, Woertler K. Measurements and classifications in musculoskeletal radiology. In: Measurements and Classifications in Musculoskeletal Radiology. 2014; Pp:59-83. doi:10.1055/b-002-91661

53. Younger AS, Sawatzky B, Dryden P. Radiographic assessment of adult flatfoot. Foot Ankle Int. 2005;26(10):820-825. doi:10.1177/ 107110070502601006

54. Kêkê LM, Samouda H, Jacobs J, et al. Body mass index and childhood obesity classification systems: a comparison of the French, International Obesity Task Force (IOTF) and World Health Organization (WHO) references. Rev Epidemiol Sante Publique. 2015;63(3):173-182. doi:10.1016/j.respe.2014.11.003

55. Evans AM, Rome K, Peet L. The foot posture index, ankle lunge test, beighton scale and the lower limb assessment score in healthy children: a reliability study. $J$ Foot Ankle Res. 2012;5(1):1. doi:10.1186/ 1757-1146-5-1

56. Zuil-Escobar JC, Martínez-Cepa CB, Martín-Urrialde JA, GómezConesa A. Reliability and accuracy of static parameters obtained from ink and pressure platform footprints. J Manipulative Physiol Ther. 2016;39(7):510-517. doi:10.1016/j.jmpt.2016.07.005

57. Reel S, Rouse S, Vernon W, Doherty P. Reliability of a two-dimensional footprint measurement approach. Sci Justice. 2010;50:113-118. doi:10.1016/j.scijus.2009.11.007

58. Akobeng AK. Understanding diagnostic tests 1: sensitivity, specificity and predictive values. Acta Paediatr Int J Paediatr. 2007;96 (3):338-341. doi:10.1111/j.1651-2227.2006.00180.x

59. Akobeng AK. Understanding diagnostic tests 2: likelihood ratios, pre- and post-test probabilities and their use in clinical practice. Acta Paediatr. 2007;96(4):487-491. doi:10.1111/j.16512227.2006.00179.x 


\section{Publish your work in this journal}

The Journal of Multidisciplinary Healthcare is an international, peerreviewed open-access journal that aims to represent and publish research in healthcare areas delivered by practitioners of different disciplines. This includes studies and reviews conducted by multidisciplinary teams as well as research which evaluates the results or conduct of such teams or healthcare processes in general. The journal

covers a very wide range of areas and welcomes submissions from practitioners at all levels, from all over the world. The manuscript management system is completely online and includes a very quick and fair peer-review system. Visit http://www.dovepress.com/testimonials. php to read real quotes from published authors. 